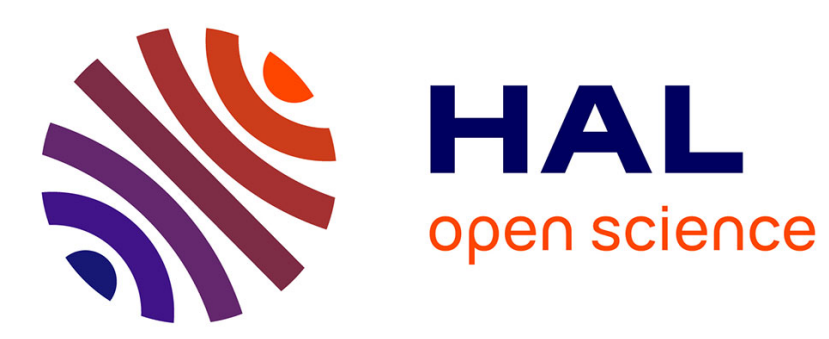

\title{
Unfolding in the Empirical Sciences: Experiments, Thought-experiments and Computer Simulations
}

Rawad El Skaf, Cyrille Imbert

\section{To cite this version:}

Rawad El Skaf, Cyrille Imbert. Unfolding in the Empirical Sciences: Experiments, Thoughtexperiments and Computer Simulations. Synthese, 2013, 169 (3), pp.557-574. 10.1007/s11229-0120203-y . hal-01098486

\section{HAL Id: hal-01098486 https://hal.science/hal-01098486}

Submitted on 25 Dec 2014

HAL is a multi-disciplinary open access archive for the deposit and dissemination of scientific research documents, whether they are published or not. The documents may come from teaching and research institutions in France or abroad, or from public or private research centers.
L'archive ouverte pluridisciplinaire HAL, est destinée au dépôt et à la diffusion de documents scientifiques de niveau recherche, publiés ou non, émanant des établissements d'enseignement et de recherche français ou étrangers, des laboratoires publics ou privés. 


\title{
Unfolding in the empirical sciences: experiments, thought experiments and computer simulations
}

\author{
$<$ version before final corrections $>$ \\ $<$ Please use the published version when citing or quoting $>$
}

Skaf, Rawad El, et Cyrille Imbert. « Unfolding in the Empirical Sciences: Experiments, Thought Experiments and Computer Simulations ». Synthese 190, $\mathrm{n}^{\circ} 16$ (1 novembre 2013): 3451-3474. doi:10.1007/s11229-012-0203-y.

Figures, unless inserted in the text, are attached as separate files or copied at the end of the document.

\section{Table of contents}

I. Introduction

II. A common description

1. Question-oriented activities

2. Scenarios

3. Unfolding of scenarios

4. Result of scenarios

5. The scientific conclusion(s).

III. Illustration of the concepts: a threefold case study

1. Smoluchowski's (1912) and Feynman's (1963) thought experiments

3. The computer simulation: Skordos and Zurek (1992)

4. The experiment: Kelly and alii (1997)

IV. Discussion of the general claim in light of the case study

V. Further evidence: solving problems and exploring physical situations in fluid dynamics

VI. Unfolding as a general task of science

1. Explicatory and strongly referential achievements

2. CS, models/representations and unfolding

3. TE as unfolders

4. Experiments as unfolders

5. Unfolding in usual practices.

VII. Functional but not epistemological substitutability

VIII. Conclusion 


\begin{abstract}
Experiments, computer simulations and thought experiments are usually seen as playing different roles in science and as having different epistemologies. Accordingly, they are usually analyzed separately. We argue in this paper that these activities can contribute to answering the same questions by playing the same epistemic role when they are used to unfold the content of a well-described scenario. We emphasize that in such cases, these three activities can be described by means of the same conceptual framework - even if each of them, because they involve different types of processes, falls under these concepts in different ways. We further illustrate our claims by presenting a threefold case study describing how a thought experiment, a computer simulation and an experiment were indeed used in the same role at different periods to answer the same questions about the possibility of a physical Maxwellian demon. We also point at fluid dynamics as another field where these activities seem to be playing the same unfolding role. We analyze the importance of unfolding as a general task of science and highlight how our description in terms of epistemic functions articulates in a noncommittal way with the epistemology of these three activities and accounts for their similarities and the existence of hybrid forms of activities. We finally emphasize that picturing these activities as functionally substitutable does not imply that they are epistemologically substitutable.
\end{abstract}

Key words: Experiment - Computer simulation · Thought experiment • Unfolding • Scenario · Maxwell's demon · Physical demon - Analytic versus synthetic knowledge · Formal versus empirical disciplines $\cdot$ Functional substitutability $\cdot$ Epistemological substitutability 


\section{Introduction}

Experiments, computer simulations and thought experiments (hereafter E, CS and TE) are traditionally assigned different roles in scientific activity. For example, TE are often seen as ways of exploring conceptual apparatus and developing theorizing (Kuhn, 1964), and CS as ways of providing theoretical explanations or making predictions, which $\mathrm{E}$ hardly contribute to. Conversely, in the empiricist tradition, E are primarily considered as being the evidential source to confront theoretical representations with. Typically, in his review article about experiments in physics, Allan Franklin describes how they can be used to select or elaborate representations about the world by confirming or refuting theories, providing evidence for new entities, or helping scientists articulate theories; and when there is still no theory in the background and E seem to have a life of their own, observation are said to be "waiting for, or perhaps even calling for, a theory".

Such a description is no doubt in part correct. Yet, in the last two decades, various authors have convincingly highlighted that the boundaries between activities like modeling, experimenting, simulating or thought experimenting are not always so sharp. Morgan (2003) in particular emphasizes that scientists refuse the cuts between these activities, e.g. when talking of "using non material methods to produce observation"; more importantly, she presents cases of hybrid activities lying between mathematical models and material laboratory experiments and not fitting traditional categories (232). One may go further and note that scientists claim to be sometimes using them interchangeably to solve problems:

"Approaches to the solution of fluid dynamics problems can be classified as analytical, experimental and computational. In analytical fluid dynamics, one uses the tools of mathematics, physical modeling, and physical insight to develop solutions to engineering problems. [...] In experimental fluid mechanics, the tools include wind and water tunnels [...] the fluid "solves" the problem by assuming a certain flow state [...]. < In computational fluid mechanics $>$ the digital computer is used to solve approximate forms of differential equations of fluid motion (Gerhard and Gross, 1985, pp. 31-32).

Acknowledging these facts is one thing; but they remain compatible with a large spectrum of positions that disagree about their analysis, significance and explanation. For example, Morgan (2003) argues that CS often qualify as E, though of a nonmaterial kind (whatever this may be). Parker (2011) argues on the contrary that CS are first and foremost material experiments in a straightforward sense. Guala $(2002,2005)$ disagrees and highlights the ontological difference between E and CS. Barberousse and alii (2008) also note the different status of materiality in E and CS and argue that they differently provide epistemologically different data. As to TE and E, they are regarded as fundamentally different by those empiricists that sharply separate a priori and $a$ posteriori knowledge; but Gooding claims that TE, though conducted in mental laboratories, are just another form of experimental reasoning. Finally, Nersessian describes TE as mental simulations (but is not completely clear about the link between this notion and that of CS). Bedau (1998) goes further and claims that simulations in artificial life are TE. As to Di Paolo and alii (2000), they more cautiously indicate that CS can play the same role of questioning theoretical frameworks.

Overall, while studies comparing CS, TE and E have kept developing, the literature offers more a battlefield then a steadily developing domain; in particular, it is not completely clear how much the described similarities between these activities are deep or shallow and merely rhetorical (Cooper, 2005). More annoyingly, many of these 
disagreeing accounts heavily lean onto incompatible conceptions of these activities, which does not help disentangling these issues. In any case, does acknowledging deep epistemological similarities, existing hybrid forms and partial scientific substitutability forces us into a fusion of the corresponding categories as the only explanatory option?

Our purpose in this paper is to emphasize that CS, TE and E can be used to fulfill some basic but central epistemological function, namely the unfolding of scenarios; that because of this, they can sometimes be used with the same role within scientific activity; that when it is the case, they can be described as instantiating a common structure; that this matter of fact can be used to account for some of the similarities noted between them; and finally, that this functional position is weakly committal about the nature of these activities because scientific functions can be differently performed by genuinely different processes. This argument, though not committed to a pragmatist position, comes in a perspective that, while acknowledging the importance of ontological differences between scientific productions (experiments, theories, models), emphasizes that it is also crucial to focus upon the identification of functions, uses (Morgan and Morrison, 1999, ch. 2) or types of activities (Chang, 2011) in order to understand how science works.

The paper is organized in the following way. In section 2 we introduce some concepts that provide a common characterization of CS, TE, E as well as their different functional steps and show how these different activities can each be used for the same inquiry. In section 3, we present a threefold case study that provides a clear-cut example of how TE, $\mathrm{E}$ and CS can be used to answer the same scientific questions by unfolding the same scenario. Section 4 is devoted to discussing our claims in light of the case study. We then present in section 5 more evidence to support the general character of our claims. In section 6 , we analyze at more length the notion of unfolding as a general scientific task and discuss how our claim relates to the epistemology of CS, TE and E. We finally claim in section 7 that functional substitutability is to be distinguished from epistemologically substitutability.

\section{A common description}

Comparing models and experiments, Morgan $(2005, \S 2)$ suggestively notes that "experiments and models have much in common in the way they are used in economics" (our emphasis). In (Morgan, 2003), she also describes examples of modeling and experimenting as acts of an identical type, namely manipulations or interventions. We want to make a step further by claiming that TE, CS and E are sometimes functionally 1 substitutable. Accordingly, we now provide a functional description so as to make clearer how these different activities may perform a common role.

1. Question-oriented activities. Scientific research involves various activities such as studying material systems, producing computations, devising arguments, creating representations, etc. To the extent that research is a goal-oriented activity, these activities can be seen as means for finding justified answers to scientific questions of a theoretical or empirical nature. Answering these questions often involves providing a description of what we call a scenario (see below), investigating its content and then using the results to answer the original question.

\footnotetext{
${ }^{1}$ By "function", we mean "well-defined task that can be performed", in a sense closer to computer science or engineering than biology or sociology.
} 
2. Scenarios. A scenario is a description of a real or imaginary situation and in particular of its dynamics, which may include physical laws, rules followed by characters or external interventions on variables. The scenario usually stages different kinds of particulars, from natural objects to artificial instruments or fictional characters (such as Maxwell demon) and includes the depiction of a target system and a measuring apparatus in some particular configurations and initial states. Strictly speaking, scenarios are type-descriptions because they involve more or less information about the situations they depict. They may range from detailed mathematical models to vague descriptions of situations and whether the scenario is sufficiently specific to univocally answer the investigated question is already a scientific question. They may also describe situations different from those the investigation is about: one may for example explore 3 -sex biology to get insights about 2-sex biology or stage imaginary creatures like Maxwell demon to understand better our world.

3. Unfolding of scenarios. Scientific achievements usually require investigating the content of these scenarios in accordance with their dynamics. Such investigation can be made in various ways. One may provide a mathematical treatment in terms of equations and solve them analytically or numerically; one may also use informal arguments or resort to various types of scientific reasoning, like dimensional analysis, analogical reasoning, asymptotic or statistical analysis; or one may observe a physical situation instantiating this scenario. These different means of exploration involve various types of processes: some purely physical, some described by algorithms and computers, some mental. We call these processes "unfolding processes" since in each case, what is involved is a process the role of which is to unfold, so to speak, the content of the original scenario, which was originally "packed" in its original description and was not epistemically accessible.

It is crucial to distinguish the target processes that are described within the scenario, such as the physical evolution of the particulars involved, or the manipulations carried upon them, from the actual unfolding processes (hereafter U-processes), such as the running computer or the mental activity of the TEer, that are used to investigate the target processes and unpack the content of the scenario.

4. Result(s) of scenarios. If the U-process is successful, it provides about ${ }^{2}$ the investigated scenario nontrivial pieces of information, which can be used to bring elements of answer to the original question. Sometimes, this output is simply the final state of the system described within the scenario but it can be less precise, like in MonteCarlo simulations, in which only average quantities are physically meaningful. Further, heavy pieces of "internal" argumentation about the scenario and the validity of its unfolding may be required to determine what this result exactly is, especially when what is being unfolded is slightly different from the target scenario - because idealization, approximations, averaging effects or compensating mechanisms are for example involved.

5. The scientific conclusion(s). The result of the scenario is not to be confused with the scientific conclusion that is reached, which should be an answer to the investigated

\footnotetext{
2 In other words, the unfolding provides what Barberousse and alii (2008) call data about target situations (though these are not necessarily data from empirical origin).
} 
question. It is arrived at by taking into account the description of the scenario, how it was unfolded, its result, and by providing some arguments showing the implications for the original question.

Obviously, the different steps described above do not provide complete definitions of $\mathrm{CS}$, TE or E and for this reason cannot offer specific insights into their epistemology. Their intended virtue lies in the fact that they offer a description, obtained through philosophical abstraction, which is poor enough to be common to these activities, beyond their differences, but yet sufficiently informative to provide a framework to analyze them together and draw non trivial consequences about their use in science. The claim underlying this common description is that E, TE and CS can be seen as composed of functionally similar parts, and that, beyond their deep differences, they sometimes play the same scientific role. When they do, we shall say for short that they fit a CUI pattern of inquiry (construction of a scenario in the context of an inquiry, unfolding of the scenario, interpretation of the result).

\section{Illustration of the concepts: a threefold case study}

Exhibiting a common abstract description of some scientific activities is not a sufficient indicator that, from a scientific point of view, they perform the same type of activity: volcanoes expulse gazes and state ambassadors, but nobody claims that states and volcanoes should be analyzed together as performing the same activity of expulsion. More compelling evidence is provided if we find a case in which a CS, a TE and an E are alternatively used to answer an identical scientific question by unfolding an identical scenario. We now present a threefold case study illustrating this possibility.

The second law of thermodynamics, formulated by Clausius and Thomson in the mid 19 th century, says that it is impossible for heat to pass from a colder to a warmer body unless some other change accompanies the process. This version of the second law was supposed to apply to all thermal phenomena at the micro and macro level. It was further believed that this universal scope could be explained by grounding the second law on the laws of mechanics. Maxwell was an atomist and the inventor of the kinetic theory of gases, which defines heat through the velocity of molecules. For him, if the second law was to be founded on the microscopic constituents of matter, then it had to be a statistical law. This is why he presents in 1867 his famous TE. Let us conceive a very observant and neat fingered finite being that can follow every molecule in its course. Then let us suppose a vessel full of air at equilibrium divided into two portions $A$ and $B$ by a division in which there is a small hole. If that being, later known as Maxwell demon, opens and closes this hole so as to let only the faster molecules pass from A to B and the slower from B to A then the demon will violate the second law of thermodynamics, which cannot therefore be universal.

In the early 20th century, it had then become clear that thermal processes were statistical processes. Empirical violations arose in observable fluctuation phenomena, such as Brownian motion of a pollen grain. But could these microscopic violations be used to accumulate work and produce macroscopic violations? The investigation then turned into a search for physically possible demons instantiated by purely mechanical devices.

As we shall now see, this research was lead through thought experimental, computational and finally experimental means in the late 20th century. In each case, the 
investigation was meant to answer (among other goals) the following questions: Q1: Can the existing microscopic violations of the second law be physically accumulated to produce macroscopic violations?; Q2: If not, what is the explanation of this failure? And in each case the inquiry went through the unfolding of a common scenario staging situations with a physical asymmetry in the apparatus, which, in macroscopic circumstances, would produce asymmetric behavior.

\section{Smoluchowski's (1912) and Feynman's (1963) thought experiments}

To find a physical substitute for the demon, Smoluchowski's idea is to divide the chamber with a trapdoor made asymmetric by a spring. The molecules should then accumulate in one chamber and create a density difference, which can be transformed to work in violation of the (statistical, or any form of the) second law of thermodynamics (figure 1).

It is hard to see why the mechanism cannot work as a perpetual motion machine of the second kind producing work for free. Nevertheless Smoluchowski concludes by means of a simple TE that no work will be produced. Here is how he unfolds this version of the scenario. Since the trapdoor mechanism is in the same isolated container as the gas and needs to be light enough to allow molecules to pass through, it will reach a high thermal energy; thus, the trapdoor will be agitated by its own Brownian motion. Therefore, the trapdoor will open without any collision and let the passage of molecules in the wrong direction and this mechanical demon will fail to work as intended.
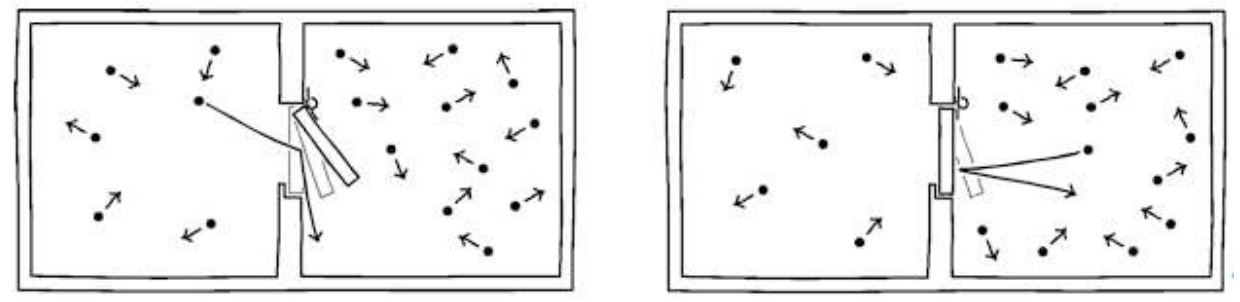

Figure 1: Smoluchowski trapdoor (Picture taken from John Norton, " Eaters of the lotus: Landauer's Principle and the Return of Maxwell's Demon" (Norton, 2005)

A very close TE, based on a germane mechanism, "the ratchet and pawl" (figure 2) is presented by Feynman (1963). Feynman's analysis is lengthier and clearer than Smoluchowski's but both keep the same spirit. The bombardment of particles in the right container pushes the vane in both directions, but because of the ratchet and pawl in the left container, the mechanism should move in one direction and produce work. Feynman nevertheless unfolds this version of the scenario like Smoluchowski. In order to stop the pawl movement after it comes off a tooth, "an essential part of the irreversibility of our wheel is a damping or deadening mechanism which stops the bouncing [of the pawl]". This results in dissipation of energy in form of heat and the pawl and ratchet also has Brownian motion. Therefore, "every once in a while, by accident, the pawl lifts itself up and over a tooth just at the moment when the Brownian motion on the vanes is trying to turn the axle backwards. And as things get hotter, this happens more often" (Feynman 46-1,2). By assuming a canonical distribution function, Feynman then shows by a brief calculation that there is on average no net work done. 


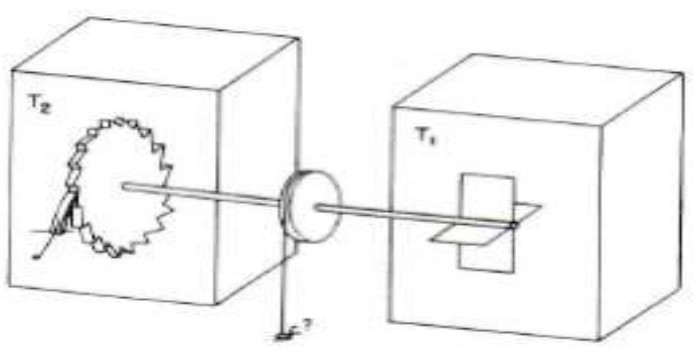

Figure 2: Feynman's Ratchet and Pawl

In brief, both Smoluchowski and Feynman investigate a promising version of the general scenario and by imagining possible effects and pointing at their interplay, they describe how the systems involved behave. Their TE also suggests elements of explanations for this behavior. To put it with Smoluchowski's words: "a perpetual motion machine would only be possible if one could construct a one-way valve of quite another kind, without a tendency to molecular fluctuations, and for that today we see no possibility." Because this explanation is not specific to the investigated versions of the scenario, a general negative answer about the possibility of macroscopically violating the second law is suggested.

\section{The computer simulation: Skordos and Zurek (1992)}

Four decades later, the development of computational resources made possible to simulate the molecular evolution of a gas. Skordos and Zurek's perform "a computer simulation of a trapdoor mechanism inspired by Smoluchowski's idea" (1992, p.94), but choose a version of the scenario adapted to computational exploration. The simulation is two-dimensional and the gas molecules within the vessel are billiard balls. All "collisions conserve energy and momentum, except for particle-wall collisions and collisions at the edge of the trapdoor, the box being assumed to be infinitely massive. The collision forces are derived from infinite hardcore potentials, and angular momentum (spin of the billiard balls) is not included in the model." The trapdoor moves horizontally between two door stops, one in the middle of the container, the other in the chamber on the left. This gives the trapdoor the same asymmetry as Smoluchowskis': it opens for molecules coming only from the right to the left parts of the container.

The results of the simulation "show that the operation of the trapdoor is consistent with the second law of thermodynamics in the sense that the particles are distributed uniformly in the available area on the average $[\ldots]<$ The $>$ simulations have also shown that the time-averaged temperature is the same in each chamber and equal to the temperature of the trapdoor [...] <and > that the time-averaged velocities distribution [...] is consistent with the Maxwell- Boltzmann distribution law and equipartition of energy" (p.99).

On this basis, Skordos and Zurek conclude that "these results confirm Smoluchowski's insight that [...] the trapdoor [...] cannot extract useful work from the thermal motion of the molecules when left on its own" (p.95). An advantage of this CS over the TE is that these results are obtained without using statistical physics (which Feynman partly relies upon) because the "purely mechanical, automated Maxwell's demons are completely described within a theoretical framework such as Newtonian physics" (p.95).

\section{The experiment: Kelly and alii (1997)}


For a long time, E involving microscopic apparatus were not possible. In the context of developing nanotechnologies, scientists started creating and controlling movements at the micro level by devising molecular brakes (1994) or rotors (see Kottas and alii 2005 for a review). While exploring rotary motion, Kelly and alii were led to build molecular ratchets and this paved the way for the experimental investigation of candidate physical demons. Their 1997 and 1998 papers describe how they synthesize and study molecules composed of different benzene rings and behaving as possible parts of a ratchet (figure 3): a toothed ratchet wheel, a pawl preventing unintended rotation and a spring holding the pawl in place.

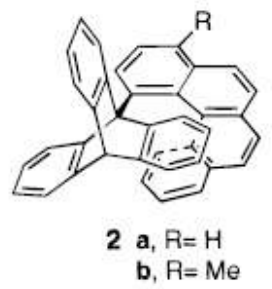

Figure 3: A triptycene-helicene molecule

Because of the molecule configuration, rotation pushes the helicene part out of planarity towards an energy maximum. The important point is that, because of a twist in the pawl, doing a rotation is supposed to be easier in the clockwise than counterclockwise direction, which is confirmed by the asymmetric profile of the variation of the energy in function of the angle out of planarity (figure 4):

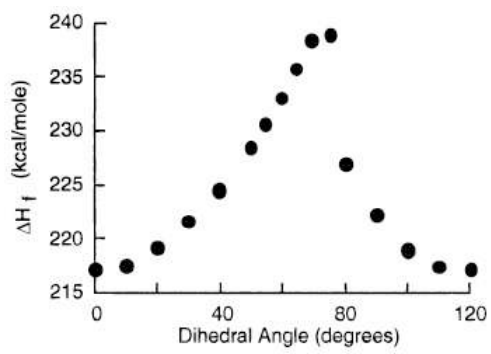

Figure 4: variation of the energy in function of the angle out of planarity

Yet, the running of the experience (unfolding step) reveals that, in this physical situation, the triptycene molecule involved in the ratchet equally rotates clockwise and counterclockwise. When interpreting the experience, Kelly and alii suggest that the explanation is that "the two energy minima flanking the energy maximum are equal in energy": "unlike mountain climbing, it is only the height of the summit, not the steepness of the ascent, that matters" $(1998,3661)$. Put another way, the principle of microscopic reversibility rules". The E illustrates "the peril of extrapolating from macroscopic to molecular scales" (1997, 1868). As they themselves note $(1998,3661)$, this experimental result and its discussion is in agreement with the more theoretical thermodynamics-based analysis by Feynman.

\section{Discussion of the general claim in light of the case study}

We do not mean to underscore the differences between CS, TE, and E - quite the contrary. What we claim is that beyond their deep differences, these activities can sometimes be different ways to perform the same task. Let us now examine in the light 
of the case study, how much these differences and the possible departures from the general characterization given above may threaten our claim.

Let us first note that the CS, TE and E described above are presented by their authors as independent ways to try to answer an identical scientific question - and not as activities differently contributing to a common task (they were carried out at different times). The CS is not in need of confrontation with empirical data nor has the $\mathrm{E}$ a life of it own because it is waiting for a theory. Each provides its own insights and epistemological credentials about one question. Kelly and alii answer Q1 by exhibiting negative experimental evidence in cases in which a positive answer may have been hoped for; the interpretation of the E provides in turn an answer to Q2. Smoluchowski's and Feynman's discussion of the ratchet and pawls highlights one aspect of the scenario (Brownian motion) that explains why accumulation of work must fail; they thereby answer Q1 and Q2 in the same time. Zurek's CS answers Q1 in the negative and provides various numerical evidence in favor of Smoluchowski's hypothesis.

We more precisely claim that the E, CS and TE are functionally substitutable in the sense that they "share" a common scenario, which they investigate by different means. This point requires clarification since it is by no means obvious to see what is exactly shared and how it is shared: E, CS, and TE are not objects of the same type (e.g. algorithms) differently performing a function that some objects of this type perform (e.g. computing a function). So can it really be said that they do the same thing?

Let us first show how ontological differences are not a worry. As noted above, a scenario presents the description of particulars of a certain type. An E instantiates the scenario by staging a particular physical system, which is a token of the scenario and which complete description is more replete than what the scenario says. A CS makes a computation the outputs of which provide information about a discrete computable model $B$ (ideally a discrete subtype of the scenario) which in turn is informative about the content of the scenario. As to TE, they rely upon mental processes involving some mental representations of a situation, which corresponds to a subtype of the scenario ${ }^{3}$. Given these differences, it is clear that these activities entertain a different relation to the scenario. So they do not share it in the sense that different co-owners entertain the same property relation to some good but in the sense that an owner of a house and the holders of the lease are in different relation with the same entity. Further, if they are used to unpack a target scenario and bring an answer to the original question, all these U-processes need, at least in principle, to be accompanied by some (functionally equivalent) pieces of (deductive, ampliative, statistical, etc.) scientific reasoning that explain why the unfolding of a replete token or a subtype of the scenario can be believed to be informative about the scenario and why the picturesque particulars in the TE do not spoil its informativeness. This said, the module composed of the unfolding processes and the arguments establishing their internal validity bring information about the scenario and can therefore play the same role.

Second, because unfolding scenarios is far from being a straightforward activity, it is submitted to numerous practical constrains. In CS, a basic constrain is tractability and this usually requires using simplifications, approximations or idealizations. For example, in the above CS, the spin of the billiard balls is not included in the model, the collisions between particles and walls do not conserve momentum and the collision forces are derived from infinite hard core potentials. In E, one needs to find systems that one knows how to manipulate, isolate from perturbation and make measurement on, which

3 Mental representations cannot include all information about a particular situation so they also correspond to types. 
leads Kelly and alii into choosing these particular molecules. And in TE, the imagined situation should be appropriate for mental reasoning. Thus, what is actually being unfolded usually is a proxy convenient version of the scenario. More differences are therefore to be expected between the actually unfolded versions of the scenario and additional specific pieces of scientific reasoning will be necessary in each case to argue that the information obtained about the proxy versions reflects what is the case in the target scenario. But again, while this makes clear that the working of the unfolding module is different for each of these activities, this do not threaten the claim that the same function of unfolding of a common scenario is performed. Birds and plane need not have identical inner workings to fly nor brains and computers to compute.

\section{Further evidence: solving problems and exploring physical situations in fluid dynamics}

The above case study was aimed at bringing compelling evidence for the existential claim that there are cases in which CS, TE and E can be used to answer the same question by unfolding the same scenario. Now, while granting the claim, the reader may be legitimately worried about its scope and suspect that the situation presented above merely applies in some rarefied context and is little informative about actual scientific practices.

Let us start with an uncontroversial point. It is indeed difficult to find inquiries in which some CS, TE, E are concurrently used in the very same role - but we do not claim the contrary. Being able to perform CS, TE, E or to solve models obey different constraints. Thus epistemic circumstances in which they can be optimally performed concurrently may be rare. It can therefore be expected that only the most appropriate route is usually used and cases like our case study, though philosophically important, are not frequent. In our example, it so happens that, because of the way that scientific technology evolved, the TE was made first, then the CS and finally the E and the three activities were separately carried out over almost one century.

It may however be the case that CS, TE or E are routinely used for different unfolding tasks within some domains of inquiry. It seems to be the case in fluid dynamics, in which unfolding is a major task and is performed, depending on the circumstances, computationally, experimentally, by solving models or by other forms of human-made reasoning possibly including TE. To comfort this view, we now present how different specialists of this field view their investigations.

We have cited in the introduction a general handbook describing analytical, experimental and computational fluid dynamics as different approaches to solve engineering problems (Gerhard and Gross, 1985). Talking of E as "solving" an equation is of course a metaphorical way to describe things, but it clearly highlights the fact that $\mathrm{E}$ may in practice play the same general role as mathematical methods. One may however suspect this quote of presenting a biased view of the role of $E$ in fluid dynamics. But Experimental Fluid Mechanics, which was for decades a major reference in the experimental part of this field, agrees:

"It is usually possible to formulate the differential equations describing the variation of velocity, pressure, temperature and other quantities of interest [...] Unfortunately, it has no been possible to solve, the equations in general [...]. Although the flow around aerfoils and other so-called streamline bodies can be adequately if laboriously calculated by choosing appropriate simplifications or approximations in different regions of the flow, even these "patchwork" 
calculation frequently require the use of empirical formulae. Accordingly, experimental fluid mechanics is a flourishing science, important both in its own right as of method of solving flow problems with or without theoretical support, and also as a source of information for the improvement of theoretical analyses." (Bradshaw, 1964 2-3, our emphasis)

Finally, the picture given by computational fluid dynamicists also concurs:

"For most of the 19th and 20th centuries there were two approaches to the study of fluid motion: theoretical and experimental. But today, because of the power of modern digital computers, there is yet a third way to study fluid dynamics: computational fluid dynamics. " (J. M. McDonough, 2009)

Even without going in the details, it is clear from the quotes that computational, experimental and theoretical methods are used as possible alternate ways of exploring the behavior of fluids having well-established mathematical descriptions but still remaining opaque. This agreement in the quotes is all the more striking as it comes from authors involved in different approaches of this field and remains from the 60ies onwards while fluid dynamics underwent a significant change in methods - since much of the activity in the field rapidly became of a computational nature. Overall, the functional substitutability between these activities also explains why experimental fluid dynamics, which was a flourishing discipline tend to be in part replaced by computational fluid dynamics.

\section{Unfolding as a general task of science}

We have argued so far that CS, TE and E can play the same unfolding role and fit what we called a CUI pattern of inquiry. We shall now analyze at more length this notion of unfolding and see how our claim articulates with the epistemology of CS, TE and E.

\section{Explicatory and strongly referential achievements}

Putting the emphasis upon the notion of unfolding, qua general scientific achievement, is a way to distinguish two kinds of valuable tasks that are performed in the empirical sciences. First, in strongly referential achievements, one tries to build radically new appropriate $^{4}$ representations of the nature of physical systems. "Radically new" means that one describes how some physical systems work by offering a representation having an original logical content and ascribing the system some unprecedented set of properties. "Appropriate" may be interpreted, depending on one's epistemological position, as "true" or "empirically adequate": in both cases, the task is one of making a felicitous act of reference about some partly unknown otherwise designated physical system to which we have no straightforward epistemic access and the difficulty lies in bridging this referential gap between representations and their worldly target objects. This is the kind of achievement that is performed by new theories and to which radically new observations contribute when they are used in an evidential role as constrains upon the search for hypotheses. Should we believe that we possess a complete true description of some system A, then this type of activity about A would stop because no radically new act of felicitous reference and ascription of novel physical content to these systems would be believed to be possible. When taking this endeavor as the primary scientific activity, E are then analyzed as ways of providing evidence in order to confirm, 
falsify, or guide the choice or refinement of theoretical descriptions of physical systems and the claim that E, CS and TE may play the same role is hardly conceivable, since for empiricists CS and TE cannot be primary evidential sources.

Another valuable task is to investigate how some (real of fictional) physical systems of a well-described type, which theoretical description is assumed to be given, do behave because of their belonging to this type. (By "well-described", we here mean wellidentified and consistent ${ }^{5}$ ). What is then produced can be called "explicatory knowledge", because what one does is to explicate (unfold in Latin) the content of some scenario: the difficulty is to have epistemic access to the content of a given theoretical representation, that is to bridge the epistemic gap between different connected aspects of this representation. The statements presenting the gained explicatory knowledge typically say that in this or that situation, only defined by some set of properties, this or that behavior is/is not/can/cannot be met. Science is here apparently less sensational since no radical novelty is involved in what is found, which is in principle contained in the original description, and everything boils down to explicating presumably analytical statements. This is perhaps why philosophers often ignore this aspect of scientific activity, because they implicitly focus upon radical logical novelty as well as what is possible in principle and not in practice (Humphreys, 2004, 154), even if the latter gives a better picture of scientific activity.

To put it in the terms of the model theoretic view of theories, the first task is to try to identify the phase spaces of physical systems otherwise designated while the second is to explore the properties of some pre-defined phase spaces. While explicatory achievements are made by processes that are believed to unravel the content of scenarios and the corresponding knowledge is presumably analytical ${ }^{6}$, in strongly referential achievement, some potentially novel physical content is ascribed to some worldly system and it is not known whether this content follows from what we believe to be knowing about this system. So these achievements differ by the nature of the task involved and by the epistemic statements that are produced.

In this perspective, it is also crucial to distinguish between the type of activity involved and the type of task to be completed. The predicates "strongly referential" and "explicatory" used above are meant to point at some epistemic task within some inquiry and representational activities may sometimes involve some explicatory but no strongly referential tasks: when a scenario already fulfills conditions for felicitous reference (truth, empirical adequacy, etc.), it can be said to be well-established (versus simply well-described); then, making felicitous acts of reference is not a problem even if the epistemic task of explicating the content of the representation and thereby investigating the behavior of the system still remains. The activity can then be said to be weakly referential: within weakly referential activities, the nature of the object to be represented (or the in principle content of its representation) is already known even if determining how it behaves and how to represent its different aspects may still be a real question - and how to model well-known fluids is a vast field of research. In such activities, the scenario has a representational function or role but there is no strongly referential task to perform and the scientific task is to extract information out of established representations or about well-known systems.

\footnotetext{
${ }^{5}$ In some cases, identifying the physical inconsistency of a scenario may be the target of the inquiry and this condition should then be relaxed.

${ }^{6}$ For this reason, the CUI pattern might not apply to ampliative TE - unless the ampliative step is made within the interpretion I-step.
} 
So both strongly referential and explicatory tasks may be related to representational inquiries: thus representing is not a notion that is sharp enough for the present investigation- even if representing or providing representations having some specific virtues may sometimes constitute the right category to describe some scientific inquiries.

\section{CS, models/representations and unfolding}

A way to rephrase our claim is to say that explicatory achievements can be performed by CS, TE and E. Let us now see how this claim articulates with the epistemology of CS, TE, and E. Our main purpose is to show how it is coherent with (accepted versions of) them, though noncommittal about the specific nature of these activities.

Let us start with the unproblematic case of CS. The claim that CS are possible ways of unfolding scenarios comes as no surprise since this notion of unfolding has been primarily developed in the context of the analysis of computational science. As noted above, Humphreys emphasizes that, when studying science, it is crucial to keep in mind the in practice possibility of carrying out activities (e.g. computing the values of a function) and in particular of having access to the content of theories or representations; in this perspective, he argues that it is crucial to analyze dynamical representations like simulations because they are in practice ways of giving access to the hidden content of theories or models. In the same vein, Winsberg emphasizes the importance of "theory articulation" (2003), which he describes, following Kuhn, as the process of making actual the potential order provided by new theories and thereby "bringing <theories> into contact with the world". Winsberg also notes the reluctance of Kuhn to see theory articulation as a form of knowledge production, that is as a process requiring an epistemology of its own, and he conversely emphasizes that simulation techniques are used in order to learn about the behavior of these systems (2003, abstract), even if he does not characterizes which specific type of knowledge is here involved.

The fact that exploring the content of representations is not straightforward is given an even more central place by authors like Hughes, with his DDI account of scientific representation (Hughes, 1999), or Suarez (Suarez, 2004), with his inferential account of representations, since what we call unfolding is for them a constitutive aspect of scientific representation. Hughes analyzes representation as a three part notion: first the denotation of a physical system by a model; second the demonstration of the dynamical consequences of the model; third the interpretation of these consequences in terms of the physical system. Hughes even goes further when he emphasizes that the demonstration can be done by other means than mathematical ones and describes how material models can play this role: " "The internal dynamic of the mathematical model is supplied by a mixture of geometry and algebra, that of the material model by <...> natural processes. $<\ldots>$ But all these modes of representation share this common feature; they contain resources which enable us to demonstrate the results we are interested in." (Hughes, S327, 1997, our emphasis).

We are of course clearly sympathetic with these ideas. Our point of concern is simply that the activity of unfolding is here presented as a component of some referential activity, namely the articulation of theories to phenomena (Winsberg), or the activity of scientific representation (Hughes). Of course, in the empirical sciences, most - if not all scientific inquiries are meant at providing in the end some knowledge about worldly systems, so most unfolding activities can be seen as part of some representational inquiry; but, as emphasized above, such an all-encompassing way of describing science 
fails to highlight the deep differences described above between strongly referential and explicatory achievements. Further, denotation can be realized in the absence of demonstration (like in the case of scientific images) and demonstration (unfolding) can be performed in the absence of actual denotation or representation (as when one investigates a central mathematical structure such as the Ising model, because it is worth exploring for its own sake and independently of how it is applied or represents in this or that circumstance). Accordingly, the analytic philosopher should conceptually distinguish these types of activities - which does not involve a commitment about how frequently they are instantiated separately in actual science.

To put thinks differently, rich and synthetic notions of representations like Hughes', which merely describe demonstration as a component of the activity of representing and not as a general task that can be performed among other means by some representations, fail to identify unfolding as an independent task and does not help clarifying why representational (CS, TE) and non representational activities (E) may sometimes perform the same task or why hybrid forms of them can be created. In our view, the DDI account of representation could best be seen as a specification of the CUI pattern of inquiry for the case of scientific representations.

\section{TE as unfolders}

Let us now turn to TE. Because TE have been seen as a challenge to empiricism, much of the debate about them was framed by the question "How can we learn genuinely new statements about reality (if we can at all), just by thinking?" This is what Norton names the epistemological problem of TE in the sciences: "Thought experiments are supposed to give us knowledge of the natural world. From where does this knowledge come?" (Norton, 2004b, 8). In this perspective, TE are primarily analyzed as contributing to what we called referential tasks and this usually comes hands in hands with an emphasis upon radical novelty and ignorance of unfolding as a knowledge productive activity. Typically, Kuhn (this time in the context of his analysis of TE) writes: "Because it embodies no new information about the world, a thought experiment can teach nothing that was not known before" (Kuhn, 1964, 252).

In this perspective, our description of TE as scenario unfolders naturally fits with these accounts of TE that emphasize that mental reasoning is epistemically beneficial and constitute a creative part of scientific practice. Such accounts fully acknowledge that extracting information from (mental) models is a scientific achievement, even in non ampliative cases, and this is precisely where mind are sometimes good at, by their ability of making "mental simulations" by the "construction of mental models" (Nersessian, 1992, 292) or of "<manipulating> a certain kind representations" especially quasi-spatial ones (Miscevic, 1992).

This said, the functional description of TE as ways of unfolding scenarios, because it simply highlights what TE $d o$, remains neutral about how they do it and about the vexed question of what they are, while still providing some non trivial ground for analyzing their role and place in science. It is even compatible with the position that TE are arguments in disguise (Norton, 1996, 2004a, 2004b) because arguments are also a way to extract information from premises. What our functional description imposes however (and what is somewhat blurred by Norton's account) is that for TE like for CS or E, one should clearly distinguish between different parts, namely the discursive construction of the scenario in relation to a scientific problem, the unfolding (however this step is cashed out in a full-fledged account), the arguments discussing the "internal validity" of 
the unfolding (Winsberg, 2009), the arguments discussing the informativeness of the scenario result about the target physical situation ("the external validity"), and the argument tacking back the result to the original question (see for example (Gendler 1996, p.37) for a close analysis of the structure of TE).

Further, when it comes to analyzing and disentangling the similarities between TE and $E$, our purely functional and noncommittal perspective is more parcimonious. For example, Gooding (1992) adopts a heavily committal position in order to account for the similarities between TE and E. He claims that TE, though conducted in mental laboratories, do not cease to be E (281) and that their effectiveness "is largely attributable to features that they share with <real experiments>" (282), namely "instrumentation and embodied actors" having sufficient crafted representational capacity and perceptual embodied familiarity with the world to be able to perform them and benefit from their narratives. Gooding thereby gives in his analysis of the nature of these activities and of the explanation of their similarities a central importance to the capacities required to perform $\mathrm{TE}$ and $\mathrm{E}$ and to the narratives accompanying them. Pointing at a common performed epistemic function (explicating scenarios) gives a more neutral rationale for their (partly) interchangeable uses and the potentially similar properties required from the agent to perform them or enjoy their results (e.g. tacit knowledge, perceptual familiarity), while leaving open the possibility that the central Uprocesses involved may be of a radically different nature. Saying that birds and planes fly does help understand some of their similarities and why both may deliver messages while leaving open the question of whether birds are planes (if it still seems worth asking).

\section{Experiments as unfolders}

E, when successfully carried out, provide experimental knowledge about physical systems, that is, true statements describing how some unambiguously designed systems behave and obtained from a chain of causal interactions originating in these systems. Accumulating experimental knowledge is in itself a valuable achievement. Yet, simply talking of "experimental knowledge" obscures the fact that, depending on what we already know about the experimented systems and how we can refer to them, E produce different types of knowledge, and may play different functions within scientific inquiry, in particular, referential or explicatory ones. Where we can only refer to the systems involved by means of deictics ("this happened at such time and place"), proper names ("the Earth is getting hotter)", or descriptions that are sufficient for practical identification but not for analytical investigation ("dry branches from trees burn easily"), the observed behavior falls out of the scope of what the identifying procedure implies. Then the gained knowledge cannot be ascribed to a well-defined scenario but is attributed to some partly unknown $\mathrm{X}$ in the world. Such situations offer a strongly referential challenge to scientists, which usually use the gained behavioral knowledge as evidence for finding good representations of the nature of this $X$ - and it is in this perspective that $E$ are usually analyzed in the empiricist tradition.

However, saying that Kelly and alii try to meet such a strongly referential challenge would be a misdescription of their investigation and what is at stake in it. Their $\mathrm{E}$ is not presented as the evidential touchstone that should confirm or falsify some representational hypotheses provided by Smoluchowski, Feynman or Skordos and Zurek. They no doubt primarily provide experimental knowledge about some types of molecules. But these molecules already have rich theoretical descriptions and their $\mathrm{E}$ are 
not aimed at refining them nor at providing radically new information about these systems. Kelly and alii explicitly say that they are after a molecular device functioning as a ratchet - something that could lead to a violation of the second law. While they could simply try to identify physically unknown systems having the right behavior, they choose known molecules having the right properties in term of structure (helicity) and energetic barriers to rotation and use their existing knowledge about them to compute their energy landscapes (figure 4) in order to recreate in material form an adequate token of the target scenario and investigate its behavior. Accordingly, their work is best seen as unraveling how some well-described systems do behave in some circumstances. Their conclusion is that this known physical system, though exhibiting an asymmetry (key element of the characterization), does not lead to a violation of the second law (behavior). This only makes sense if the observed behavior, though not accessible by mathematical means, is believed to be covered by the provided characterization. In other words, the statements describing their results are presumably analytical even if this belief might be false because the system does not instantiate the target scenario, something has gone wrong in the $\mathrm{E}$ or the behavior is in fact not covered by the characterization.

Overall, Kelly's E serves an explicatory role about some version of the target scenario and the material system instantiating it. Like for all E, their results are also supposed to be informative about the class of similar (that is, sharing the same dimensionless description) systems and represent them, so to speak. In this sense, their results indirectly serve a weakly referential role by enriching our picture of the behavior of this class of systems, which we already have a fundamental description of. In brief, their E provides some empirical explicatory knowledge about the set of physical situations instantiating this version of the scenario and also, by means of additional inductive arguments, clues about other versions of the scenario if not about the scenario itself.

Four more remarks should be made. First, using experimental systems for explicatory achievements clearly requires that we strongly believe that we know their dynamics. Analog computer and analog simulations ${ }^{7}$ is probably the case in which this belief is strongest and we can therefore use them for purely unfolding purposes, given that we have no interest in their outputs qua information about the physical computer. In these material procedures, a physical system is chosen because its dynamics is strongly believed to instantiate some mathematical dynamical equation (which may itself be used to represent another target system ${ }^{8}$ ) and it is seen as producing solutions to it. From this point of view, even if they are now a somewhat marginal practice, the philosophical significance of analog computers and simulations is important because they illustrate in a pure form the possibility of using material systems for unfolding purposes.

Second when used in their evidential function, E can also be seen as unfolding the behavior of some system. But if we do not know how to describe theoretically the corresponding scenario and make explicit what is being unfolded, we are not in an epistemic position to use the E in an unfolding function. Should however the right theoretical description of the physical situation be known afterwards, the E could be retrospectively be seen as having unfolded the corresponding scenario.

\footnotetext{
${ }^{7}$ See Humphreys $(2004, \S 4.5)$ for a philosophical presentation.

${ }^{8}$ Scale simulations (using for example wind tunnels and scale model of aircrafts made of the same stuff) may give the false impression that the scale model directly represents the target system. But what primarily matters is that the dimensionless equations describing both systems be identical and this may sometimes imply using different stuffs (e.g. oil instead of water) to keep these dimensionless numbers equal and correctly unfold the mathematical equations - which can hardly be understoof if one supposes that the material system directly represents the target system.
} 
Third, while an empiricist should be committed to the claim that E are central activities for playing this evidential role, she may acknowledge that E can also play an unfolding role in science, especially in these fields where physical principles are considered to be uncontroversial. All it takes is to accept that $\mathrm{E}$ may be valuable for different tasks, and that unfolding is one of these. Then, in an epistemic world in which scientists would know that they possess all true theories describing all physical systems of all natural kinds, there would still be a role to be played by $\mathrm{E}$.

Finally, the use of E in their explicatory role relies upon the belief that we know which scenario is being instantiated and explains the observed behavior. But this belief may turn out to be false. Thus, E never completely lose their potential evidential function and their results might always be reused later in this role. Acknowledging this is by no means incompatible with our claim that $\mathrm{E}$ are actually used in an unfolding role. Actual scientific activities rely upon some well-entrenched beliefs many of which can still be revised - just like what counts as observation depends upon our developing revisable epistemic situation (Shapere, 1982). Overall, E can be described as presumably unfolding well-identified scenarios (this is often their actual function) with a higher or lesser probability (this is the epistemological caveat). The situation is symmetric for CS, which are said to provide data about physical systems whereas they perform nothing more than manipulations upon logical strings and do not involve interactions with these systems: CS can be used as data providers only in so far as they unfold models which are believed to refer correctly to reality (Barberousse and alii, 2008); and when these assumptions are found guilty, the so-called data are downgraded to the statute of information about a non referring model. So CS can be described as often providing data about systems (actual role) with a higher or lesser probability (epistemological caveat).

Overall, at the very least, a picture of science in which the logical distinction between analytical and synthetic statements is mirrored by the distinction between empirical and formal methods (or disciplines) and their scientific function (providing analytic or synthetic knowledge) is too coarse to describe the various actual roles played by E or CS in the empirical sciences.

\section{Unfolding in usual practices.}

We have argued so far that unfolding scenarios is an independent function that can be carried out by different performers such as CS, TE or E and have tried to present simple and pure cases of scientific activities in which one single scientific task is performed. As a result, the analysis presented was somewhat one-dimensional. Now, though philosophically important, pure cases are rarely met in practice. Let us now see how unfolding may take place in more intricate ways.

First, unfolding ma sometimes be performed in "impure" ways: for example, in weakly referential tasks, the fact that an unfolded scenario should in the end represent some target physical situation is often used to guide and facilitate its unpacking by using information extraneous to the original scenario. This is typically what is done when physicists solve a mathematical problem by selecting "physical solutions"; or when a physical and mathematically well-described problem is solved by injecting some empirical information in it (e.g. about the observed symmetries within the phenomena).

Also, scientific inquiries are usually complex and involve performing several different epistemic tasks, in particular explicatory and referential. Nevertheless, because we provide an analysis in terms of functions and functions can be nested and combined indefinitely, this is no worry for our claim. The CUI pattern is centered around the 
scientific function of unfolding, with the constructive and interpretation steps describing why and how the scenario and its results are used within inquiries. Accordingly, instances of the CUI pattern can be plugged in complex activities described by much more intricate patterns - and conversely, the constructive and interpretative steps can be as complex as necessary and include other functional steps, including unfolding ones. Explicatory achievements may therefore be found within complex activities such as explaining, predicting, applying, testing, developing behavioral knowledge about worldly systems, or the recursive practice of model-building, in which unfolding a theoretical framework serves the generation of good representations, by going back and forth between versions of a general model and the world.

To make things clearer, one should distinguish between the function of the Uprocesses (completing some explicatory task) and the functions of their results: in the framework of elaborate inquiries, the explicatory knowledge gained by CS, TE or E is usually valuable for other uses, including representational ones. For example, when one already has supposedly some complete representations of physical systems, explicatory results can be reinjected in the building of new representations that present them through new variables, at different scales, by means of new mathematical tools, or in incomplete but more tractable ways. Take the study of fluids in domain of parameters in which they obey Navier-Stokes equations, which include some turbulent regimes. Then, as emphasized above, the unpacking of these situations can be performed by E, which "solve" the corresponding equations. "Direct numerical simulations" are another route, in which the whole range of energetic scales from the dissipation scale to the integral scale are represented (so no turbulence model with additional assumption is involved in the simulation). While these simulations are extremely costly, the explicatory knowledge they provide is crucial to develop, along with experimental information, less costly turbulent models that are reliable only about some aspects of turbulent phenomena. Here the E or CS perform some explicatory task which results are used to re-represent systems already having fundamental descriptions. In brief, this explicatory knowledge is used as "evidence" for selecting models in the space of partial and approximate re-representations of some already known system.

Practice may finally be more complex in that it provides hybrid (versus purely experimental, computational, analytical or mental) unfolding processes or activities, like those presented by Morgan (2003) (see our introduction). But the existence and development of hybrid forms is less surprising if activities involving CS, TE or E may instantiate common patterns of activities and be composed of functionally equivalent moments. Then, mixed arguments or inquiries can be expected, in which some steps may be filled by various functionally equivalent activities. The existence of cyborgan (deeply hybrid) forms of activities or processes also comes as no surprise. Simulations in which the inputs are from empirical origin or continuously fed in with updated empirical data (like in weather forecast predictions) are such a case. Another original case, met in climate science, is when the dynamical unfolding core of a CS comes from $\mathrm{E}$, for example in CS representing air particles which are drifted by winds and get dry when passing through a cold area (this is the dynamics) and the winds and temperatures are provided by empirical fields of average winds and temperature at different periods of the year (Sukhatme and Pierrehumbert, 2002).

\section{Functional but not epistemological substitutability}


We finally want to emphasize that functional substitutability does not imply epistemological substitutability and keeping this in mind is instrumental in understanding their scientific uses. We have argued that CS, TE and E, while involving different ontological types of particulars need not always serve different complementary functions; but claiming that $\mathrm{CS}, \mathrm{TE}$ and $\mathrm{E}$ can sometimes be functionally substitutable simply means that they can do the same thing, like two barristers or two goal-keepers are functionally substitutable but need not have the same talent. Similarly, CS, TE or E need not, from an epistemological point of view, play their unfolding function identically nor come with warrants or credentials of the same type, have the same degree of trustworthiness or bring the same epistemological benefits. In short, being epistemologically different activities, they are not scientifically equivalent when they are employed in the same role.

In our threefold example, each inquiry comes with its own benefits and insights. For example, Kelly and alii add some comments about the irrelevance of the shape of potentials to account for what happens at the micro level (versus at the macro level); Skordos and alii emphasize that the simulation-based insights they provide do not rely on statistical mechanics and assumptions of molecular chaos and rely on purely mechanical grounds; and Feynman explicitly says that his TE should provide an "elementary explanation" of some thermodynamics results providing some more immediate understanding (Feynman, 1977 §46.1). So, while we emphasized above that, because they obey different constraints, concomitant uses may be rare, the epistemological differences between these activities partly explains why they are not merely redundant ways of doing the same things and may still be worth performing even when their counterparts have already been carried out. This makes in particular clearer why Kelly and alii could still feel the need to perform their E, even after Skordos simulations in the 1990ies and Feynman or Skordos' TE decades before. In any case, all these CS, TE and E unfold a different version of the scenario, so each brings its own clues about the general validity of the result for the scenario itself.

So while CS, TE and E need not be complementary in the sense that they are used to perform different types of epistemic tasks, they may still be epistemologically complementary ways of performing the same task. From this point of view, it is worth mentioning that while the CS, TE and E described above were performed separately, each of these activities was built by elaborating on previous results or insights. The discussion of Skordos' CS as well as Kelly's E are explicitly reminiscent of Feynman's and Smoluchowski's arguments. Further, both the CS and the E are developed in the context of the previous TE by Feynman and Smoluchowski, which served as a first conceptual draft, motivated their inquiry and guided their investigation.

Chandrasekharan and Nersessian (2011) suggest that computational modeling may bring about the end of TE. Indeed, because CS are more reliable and elaborate activities and because our computational (and experimental) capacities keep developing, TE might be in part replaced by CS or E, like E were for a part replaced by CS in fluid dynamics. Still, the above analysis may incline to think that mental modes of reasoning may remain necessary for scientists: they may be useful to orientate within computational science because they reformulate results or insights developed by other means in human-friendly and graspable forms; they may also be ways to elaborate preliminary sketches of scientific ideas or hypotheses and serve to guide the inquiry further, like Smoluchowski's and Feynman's TE did. In this perspective, precisely because they convey different benefits and are possible in different circumstances, CS, $\mathrm{TE}$ and $\mathrm{E}$ may perpetuate as unfolding procedures in different scientific niches. 


\section{Conclusion}

When analyzing the empirical sciences, philosophers often focus upon the production of (strongly) referential knowledge, such as the discovery of true or empirically adequate theories or representations of physical systems. We have argued in this paper that producing explicatory knowledge, that is giving epistemic access to the content of well-described real or imaginary situations, is also a major task in the empirical sciences, even if it involves no radical novelty. Further, we have claimed that CS, TE and E can perform this analytic task of unfolding and can be seen, when used in this role, as sharing a common functional structure. We have also noted that this functional substitutability does not imply epistemological substitutability and thereby refined the existing analyses of the partly interchangeable role of CS, TE and E in science. We have emphasized that a correlate of our claims is that pictures of science in which analytic propositions are the objects of the formal science only and E only provide synthetic knowledge or have an evidential role are too coarse descriptions that mischaracterize scientific practice. In other words, there is no exact match between investigations about different types of statements (analytical and synthetic), types of disciplines (formal and empirical) and types of methods (non-empirical and empirical).

To illustrate and confirm our claims, we have presented a threefold case study, which stages the successive uses in identical roles of a TE, a CS and an E to answer questions about the possibility of a physical Maxwellian demon. We have also presented the possibly common role of $\mathrm{E}, \mathrm{CS}$ and theoretical human reasoning in fluid dynamics, as described by scientists of this field. These two examples illustrate that our analyses can apply both for cases that philosophers would describe as belonging to the applied sciences and (more surprisingly perhaps) for the investigation of fundamental theoretical questions.

We have also emphasized that this common analysis of CS, TE and E, while too abstract to account for the specific epistemological features of these activities, brings about philosophical benefits. It provides some grounds for analyzing some of the oft-noted similarities between these activities without committing to statements about their identities; it explains why in actual practices, these activities are alternately used and why computational methods may sometimes replace experimental ones; it finally makes the analysis of hybrid forms of science non problematic. 


\section{References}

Barberousse, Anouk, Franceschelli, Sara, \& Imbert, Cyrille. (2008). "Computer Simulations as Experiments", Synthese 169 (3): 557-574

Bedau, Mark A. (1998). "Philosophical Content and Method of Artificial Life", in T. W. Bynam and J. H. Moor, eds., The Digital Phoenix: How Computers are Changing Philosophy, Basil Blackwell, pp. 135-152, 1998.

Bradshaw, Peter. (1964). Experimental fluid mechanics, Pergamon Press: Macmillan

Cooper, Rachel. (2005). “Thought Experiments”, Metaphilosophy, 36: 328-347.

Chang, Hasok. (2011). "The Philosophical Grammar of Scientific Practice". International Studies in the Philosophy of Science 25 (3): 205 - 221.

Di Paolo, E. A., Noble, J. and Bullock, S. (2000). "Simulation models as opaque thought experiments". In: Seventh International Conference on Artificial Life, pp. 497-506, MIT Press, Cambridge, MA.

Feynman, Richard P., Robert B. Leighton and Matthew Sands. (1977). The Feynman Lectures on Physics, vol. I, original edition 1963, Addison-Wesley Publishing

Franklin, Allan. (2012). "Experiment in Physics", The Stanford Encyclopedia of Philosophy (Summer 2012 Edition), Edward N. Zalta (ed.), URL = <http://plato.stanford.edu/archives/sum2012/entries/physics-experiment/>.

Guala, Francesco. (2002). "Models, Simulations, and Experiments," in Lorenzo Magnani and Nancy Nersessian (eds.), Model-Based Reasoning: Science, Technology, Values. New York: Kluwer, 59-74.

Gendler, Tamara. (1996). Imaginary exceptions: on the Powers and Limits of ThoughtExperiment, $\mathrm{PhD}$ dissertation, Harvard University, Cambridge Massachusetts

Gerhard Philip M. and Gross, Richard J. (1985). Fundamentals of fluid mechanics Addison-Wesley Publishing

Guala, Francesco. (2005). The Methodology of Experimental Economics. Cambridge: Cambridge University Press.

Hartman, Stephan. (1996). "The World as a Process: Simulations in the Natural and Social Sciences", in: R. Hegselmann and al. (eds.), Modelling and Simulation in the Social Sciences from the Philosophy of Science Point of View, Theory and Decision Library. Dordrecht: Kluwer 1996, 77-100.

Hughes, R.I.G. (1997). Models and Representation. Philosophy of Science 64 (4): 336.

Hughes, R.I.G. (1999). The Ising model, computer simulation, and universal physics, in: Models as Mediators, M.S. Morgan and M.C. Morrison, eds., Cambridge University Press, 
Cambridge.

Humphreys, Paul. (1994). "Numerical Experimentation," in Paul Humphreys (ed.), Patrick Suppes: Scientific Philosopher, Vol.2. Boston: Kluwer, 103-121.

Humphreys, Paul (2004). Extending Ourselves: computational science, empiricism, and scientific method, Oxford University Press.

Keller, Evelyn Fox. (2003). "Models, Simulation, and 'Computer Experiments'," in Hans Radder (ed.), The Philosophy of Scientific Experimentation. Pittsburgh: University of Pittsburgh Press, 198-215.

Kelly, T. Ross, Tellitu Imanol, Sestelo José Perez. (1997). "In search of molecular ratchets", Angewandte Chemie. International edition in English, vol. 36, no17, pp. 18661868

Kelly, T. Ross, Tellitu Imanol, Sestelo José Perez. (1998). "New Molecular Devices: In Search of a Molecular Ratchet" , Journal of Organic Chemistry, 63, 3655-3665

Kelly, T. R, Bowyer, M. C., Bhaskar, K. V., Bebbington, D., Garcia, A., Lang, F., Kim, M. H., Jette, M. P. (1994). "A molecular brake” Journal of the American Chemical Society, 116, 3657-3658.

Kottas Gregg S., Clarke Laura I., Horinek Dominik, and Michl Josef. (2005). "Artificial Molecular Rotors", Chemical Review, 105, 1281-1376

Kitcher, Philip. (1993). The Advancement of Science, Oxford University Press, April

Kuhn, Thomas. (1964). "A Function for Thought Experiments", reprinted in T. Kuhn, The Essential Tension, Chicago: University of Chicago Press, 1977, 240-265.

McDonough, J. M. (2009). Lectures in elementary fluid dynamics: Physics, Mathematics and applications, lecture notes Departments of Mechanical Engineering and Mathematics, University of Kentucky, Lexington

Maxwell, James Clerk. (1871). Theory of Heat, London: Longmans, Green, and Co.

Morgan, Mary. (2002). "Model Experiments and Models in Experiments," in Lorenzo Magnani and Nancy Nersessian (eds.), Model-Based Reasoning: Science, Technology, Values. New York: Kluwer, 41-58.

Morgan, Mary. (2003). "Experiments Without Material Intervention: Model Experiments, Virtual Experiments and Virtually Experiments," in Hans Radder (ed.), The Philosophy of Scientific Experimentation. Pittsburgh: University of Pittsburgh Press, 216235.

Morgan, Mary. (2005). "Experiments versus Models: New Phenomena, Inference, and Surprise," Journal of Economic Methodology 12(2): 317-329. 
Morgan, Mary and Margaret Morrison. (1999). Models as Mediators. Perspectives on Natural and Social Science. Cambridge: Cambridge University Press.

Norton, John. (1996). "Are Thought Experiments Just What You Thought?", Canadian Journal of Philosophy, 26: 333-366

Norton, John. (2004a). "On Thought Experiments: Is There More to the Argument?" Proceedings of the 2002 Biennial Meeting of the Philosophy of Science Association, Philosophy of Science, 71: 1139-1151

Norton, John. (2004b). "Why Thought Experiments Do Not Transcend Empiricism", in C. Hitchcock (ed.), Contemporary Debates in the Philosophy of Science, Oxford: Blackwell, 44-66

Norton, John. (2005). "Eaters of the Lotus: Landauer's Principle and the Return of Maxwell's Demon Studies”, History and Philosophy of Science Part B 36 (2):375-411.

Parker, Wendy. (2009). "Does Matter Really Matter? Computer Simulations, Experiments, and Materiality”, Synthese, 169 (3):483 - 496

Shapere, Dudley. (1982). "The Concept of Observation in Science and Philosophy," Philosophy of Science, 49, pp. 485-525.

Skordos, P. A., \& Zurek W. H. (2003). "Maxwell's Demon, Rectifiers, and the Second Law: Computer Simulation of Smoluchowski's Trapdoor", in Leff, Harvey S. \& Rex, Andrew, Maxwell's Demon 2: Entropy, Classical and Quantum Information, Computing. Bristol and Philadelphia: Institute of Physics Publishing, original article in American Journal of Physics, Vol. 60, No. 10, pp. 876-882, october.

Smoluchowski, M. von. (1912). Experimentell nachweisbare, der Ublichen Thermodynamik widersprechende Molekularphenomene, Phys. Zeitshur. 13

Suarez, Mauricio. (2004). "An Inferential Conception of Scientific Representation", Philosophy of Science, 71, pp. 767-779.

Sukhatme, Jay and Pierrehumbert, Raymond. (2002). "Decay of passive scalars under the action of single scale smooth velocity fields in bounded two-dimensional domains: From non-self-similar probability distribution functions to self-similar eigenmodes", Physical Review E, 66, art. no. 056302

Winsberg, Eric. (2003). "Simulated Experiments: Methodology for a Virtual World," Philosophy of Science 70: 105-125.

Winsberg, Eric. (2009). A Tale of two Methods. Synthese, 169 (3), 575-592. 